\title{
Isomerization and Redox Tuning: Reorganizing the Maya Blue Puzzle from Synthetic, Spectral and Electrochemical issues
}

Antonio Doménech-Carbóa*, Ana María Costero ${ }^{\mathrm{b}}$, Salvador Gil ${ }^{\mathrm{b}}$, Noemí Montoya ${ }^{\mathrm{c}}$, Alejandro López-Lorente ${ }^{b}$, José A. Sáez ${ }^{b}$, Pau Arroyo ${ }^{b}$, María Teresa Doménech-Carbód

a Departament de Química Analítica. Universitat de València. Dr. Moliner, 50, 46100 Burjassot (València) Spain.

b Instituto Interuniversitario de Investigación de Reconocimiento Molecular y Desarrollo Tecnológico. Dr. Moliner, 50, 46100 Burjassot (València) Spain.

c Facultad de Ciencias de la salud, Universidad Internacional de la Rioja. UNIR, Avenida de la Paz, 137, 26006 Logroño, La Rioja, Spain.

d Institut de Restauració del Patrimoni, Universitat Politècnica de València, Camí de Vera 14, 46022, València, Spain.

* Corresponding author. E-mail: antonio.domenech@uv.es 


\section{Supplementary information}

\section{Computational calculations}

XYZ cartesian coordinates of IND, DHI and HEIND geometries optimized at RB3LYP/6-311++G(2df,2p)/vacuo level are included in this Supporting Information.

$\begin{array}{crrr}\text { IND } & & & \\ \mathrm{C} & -5.29748000 & 0.51063200 & 0.00021300 \\ \mathrm{C} & -5.18886500 & -0.88581800 & 0.00034900 \\ \mathrm{C} & -3.95659900 & -1.52948600 & 0.00024100 \\ \mathrm{C} & -2.81673500 & -0.73403300 & 0.00001100 \\ \mathrm{C} & -2.91317300 & 0.67181000 & -0.00006900 \\ \mathrm{C} & -4.15509600 & 1.29780100 & 0.00000500 \\ \mathrm{~N} & -1.48500900 & -1.11524100 & -0.00016200 \\ \mathrm{C} & -0.67759000 & -0.00029800 & -0.00022000 \\ \mathrm{C} & -1.54955600 & 1.20819700 & -0.00020300 \\ \mathrm{C} & 0.67759000 & 0.00029600 & -0.00021800 \\ \mathrm{C} & 1.54955800 & -1.20819900 & -0.00020200 \\ \mathrm{C} & 2.91317400 & -0.67181000 & -0.00006300 \\ \mathrm{C} & 2.81673400 & 0.73403300 & 0.00001500 \\ \mathrm{~N} & 1.48500700 & 1.11523900 & -0.00015700 \\ \mathrm{C} & 4.15509700 & -1.29779900 & 0.00001500 \\ \mathrm{C} & 5.29748000 & -0.51062900 & 0.00022100 \\ \mathrm{C} & 5.18886400 & 0.88582100 & 0.00035500 \\ \mathrm{C} & 3.95659600 & 1.52948700 & 0.00024500 \\ \mathrm{O} & -1.14600000 & 2.36545000 & -0.00025600 \\ \mathrm{O} & 1.14600200 & -2.36545300 & -0.00024900 \\ \mathrm{H} & -6.27544600 & 0.97045000 & 0.00028000 \\ \mathrm{H} & -6.09023600 & -1.48426600 & 0.00054300 \\ \mathrm{H} & -3.89124400 & -2.60883600 & 0.00034500 \\ \mathrm{H} & -4.21448700 & 2.37775800 & -0.00009200 \\ \mathrm{H} & -1.11119000 & -2.05166800 & -0.00003800 \\ \mathrm{H} & 1.11118700 & 2.05166500 & -0.00003600 \\ \mathrm{H} & 4.21449100 & -2.37775700 & -0.00007800 \\ \mathrm{H} & 6.27544700 & -0.97044600 & 0.00029000 \\ \mathrm{H} & 6.09023400 & 1.48427000 & 0.00054700 \\ \mathrm{H} & 3.89124100 & 2.60883700 & 0.00034700\end{array}$

$\begin{array}{crrr}\text { DHI } & & & \\ \mathrm{C} & 5.34142300 & -0.38393700 & 0.00686600 \\ \mathrm{C} & 5.15998600 & 0.95817400 & -0.31374700 \\ \mathrm{C} & 3.88005300 & 1.51496700 & -0.43034700 \\ \mathrm{C} & 2.80368500 & 0.67950400 & -0.21654700 \\ \mathrm{C} & 2.98154300 & -0.66813300 & 0.10379900 \\ \mathrm{C} & 4.23995500 & -1.21970400 & 0.22446500 \\ \mathrm{~N} & 1.42725200 & 1.04694300 & -0.28458900 \\ \mathrm{C} & 0.72391900 & -0.00053200 & -0.02862800 \\ \mathrm{C} & 1.61800600 & -1.23133100 & 0.27499500 \\ \mathrm{C} & -0.72391400 & 0.00079300 & -0.02857700 \\ \mathrm{C} & -1.61811400 & 1.23143500 & 0.27503700 \\ \mathrm{C} & -2.98159300 & 0.66814100 & 0.10380400\end{array}$




$\begin{array}{lrrr}\mathrm{C} & -2.80359500 & -0.67949000 & -0.21653700 \\ \mathrm{~N} & -1.42713600 & -1.04679000 & -0.28455000 \\ \mathrm{C} & -4.24006400 & 1.21959100 & 0.22443800 \\ \mathrm{C} & -5.34143900 & 0.38370600 & 0.00682700 \\ \mathrm{C} & -5.15986800 & -0.95839300 & -0.31376800 \\ \mathrm{C} & -3.87988300 & -1.51505900 & -0.43034600 \\ \mathrm{O} & 1.27662200 & -2.33381900 & 0.60652300 \\ \mathrm{O} & -1.27681900 & 2.33398800 & 0.60647300 \\ \mathrm{H} & 6.34295800 & -0.78303200 & 0.08773300 \\ \mathrm{H} & 6.02626800 & 1.58542400 & -0.47523200 \\ \mathrm{H} & 3.73395300 & 2.55782600 & -0.67395400 \\ \mathrm{H} & 4.36782300 & -2.26406800 & 0.47633000 \\ \mathrm{H} & -4.36805400 & 2.26394800 & 0.47628300 \\ \mathrm{H} & -6.34301500 & 0.78270000 & 0.08766800 \\ \mathrm{H} & -6.02609400 & -1.58571600 & -0.47527500 \\ \mathrm{H} & -3.73366300 & -2.55789800 & -0.67395800\end{array}$

\section{HEIND}

$\begin{array}{lrrr}\mathrm{C} & -0.00158600 & 0.00004300 & -0.00094300 \\ \mathrm{C} & -0.01329000 & -0.00005800 & 1.42438800 \\ \mathrm{C} & 1.17775400 & 0.00002800 & 2.15329300 \\ \mathrm{C} & 2.36442300 & 0.00021500 & 1.44453600 \\ \mathrm{C} & 2.39029700 & 0.00031200 & 0.03427500 \\ \mathrm{C} & 1.21971000 & 0.00022600 & -0.69311500 \\ \mathrm{H} & 1.17230100 & -0.00004800 & 3.23477800 \\ \mathrm{H} & 3.30052400 & 0.00028500 & 1.98652200 \\ \mathrm{H} & 3.34323500 & 0.00045200 & -0.47601600 \\ \mathrm{H} & 1.23134800 & 0.00029400 & -1.77410100 \\ \mathrm{C} & -1.36109000 & -0.00010400 & -0.40573900 \\ \mathrm{C} & -2.15185200 & -0.00022900 & 0.74897000 \\ \mathrm{~N} & -1.31065900 & -0.00024500 & 1.85644200 \\ \mathrm{O} & -1.83332100 & -0.00011300 & -1.65021200 \\ \mathrm{H} & -1.64513700 & -0.00029200 & 2.80520400 \\ \mathrm{C} & -3.55323000 & -0.00034300 & 0.75667000 \\ \mathrm{C} & -4.42701700 & -0.00065800 & 2.01306200 \\ \mathrm{C} & -5.63123000 & -0.00022100 & 0.05825300 \\ \mathrm{C} & -5.78739300 & -0.00003000 & 1.45680800 \\ \mathrm{C} & -6.74060000 & 0.00004200 & -0.76855000 \\ \mathrm{C} & -7.03822200 & 0.00053600 & 2.04855000 \\ \mathrm{C} & -8.00286900 & 0.00053100 & -0.16766900 \\ \mathrm{H} & -6.63090200 & -0.00006700 & -1.84399900 \\ \mathrm{C} & -8.15919800 & 0.00079200 & 1.21814500 \\ \mathrm{H} & -7.13754600 & 0.00066900 & 3.12604400 \\ \mathrm{H} & -8.88333800 & 0.00075900 & -0.79670400 \\ \mathrm{H} & -9.15145900 & 0.00117700 & 1.64657100 \\ \mathrm{~N} & -4.27482600 & -0.00046300 & -0.33985700 \\ \mathrm{O} & -4.02525600 & 0.00002400 & 3.15769000 \\ \mathrm{H} & -2.81689700 & -0.00026100 & -1.59118000 \\ & & & \end{array}$


Figure S.1. Photographic images, from left to right of: DHI, freshly prepared, unheated DHI@PAL (1 wt \%) specimen; the same specimen after 30 min; DHI@PAL P $_{180}$ specimen prepared by heating for 3 hours at $180{ }^{\circ} \mathrm{C}$ a dehydroindigo $(1 \mathrm{wt} \%)$ plus palygorskite mixture.

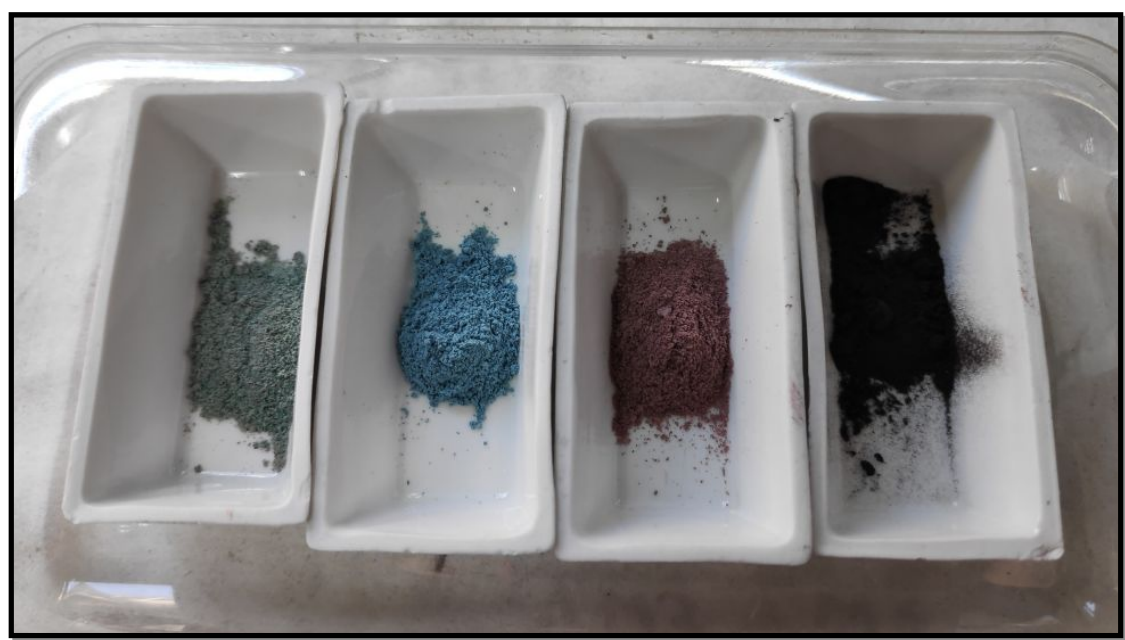

DHI@PAL 180 DHI@PAL DHI@PAL DHI 
Figure S.2. Successive uv-vis spectra recorded at a $0.1 \mathrm{mM}$ dehydroindigo solution in 0.10 $\mathrm{M} \mathrm{Bu}_{4} \mathrm{NPF}_{6} / \mathrm{DMSO}$ containing $1 \% \mathrm{v}: \mathrm{v}$ of water at intervals of $5 \mathrm{~min}$.

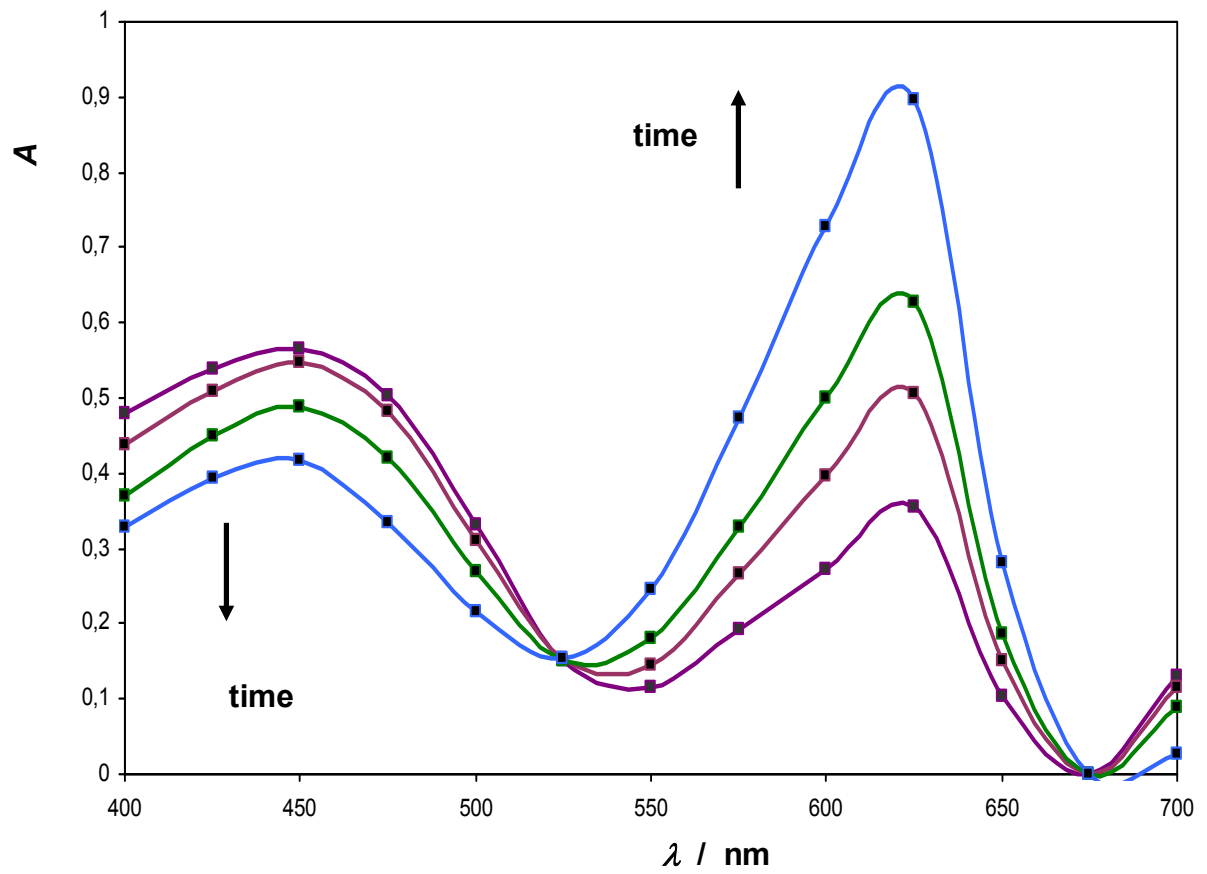


Figure S.3. ATR-FTIR spectra of a) indigo, b) IND@ $\mathrm{PAL}_{25}(10 \mathrm{wt} \%$ indigo), and c) IND@PAL $\mathrm{PA}_{160}(10 \mathrm{wt} \%$ indigo $)$. The transmittance axis is displaced for clarity.

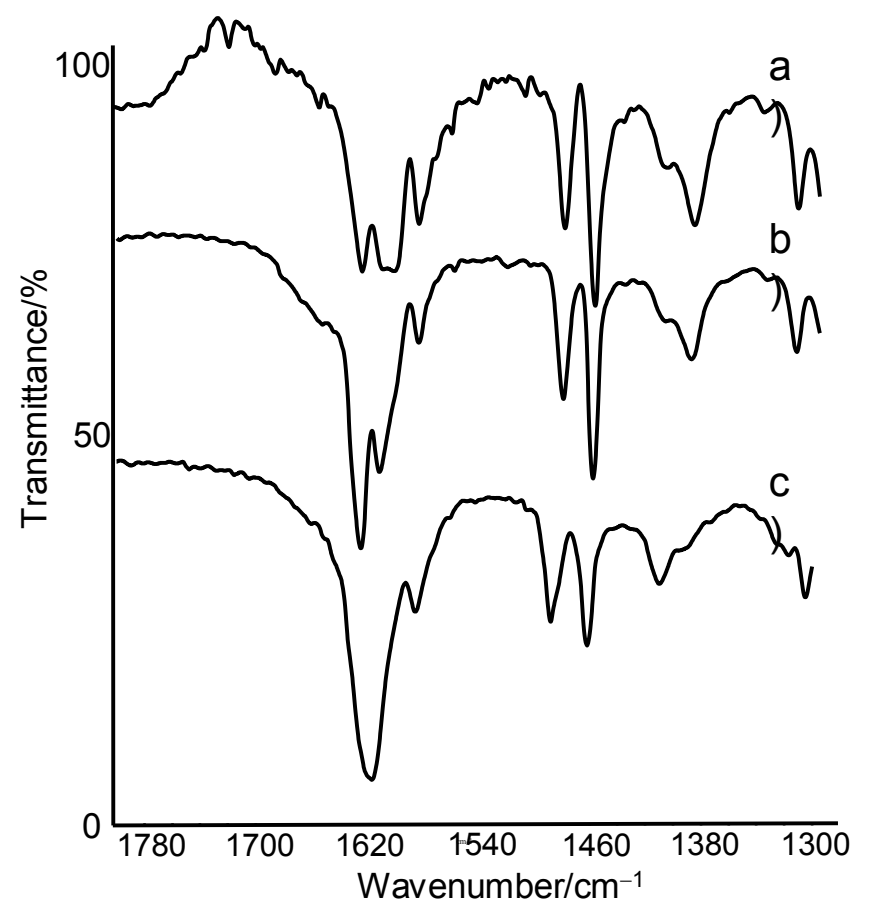


Figure S.4. Square wave voltammograms of a) indigo, b) dehydroindigo, and c) IND@ $\mathrm{PAL}_{150}$ specimen (5 wt\% indigo) attached to graphite electrodes in contact with $0.25 \mathrm{M} \mathrm{HAc} / \mathrm{NaAc}$ aqueous acetate buffer at $\mathrm{pH} 4.75$. Potential scan initiated at $-0.75 \mathrm{~V}$ vs. $\mathrm{Ag} / \mathrm{AgCl}$ in the positive direction; potential step increment $4 \mathrm{mV}$; square wave amplitude $25 \mathrm{mV}$; frequency $5 \mathrm{~Hz}$. All voltammograms show the signals corresponding to the oxidation of indigo to dehydroindigo $(0.45 \mathrm{~V})$ and the reduction of indigo to leucoindigo $(-0.30 \mathrm{~V})$.

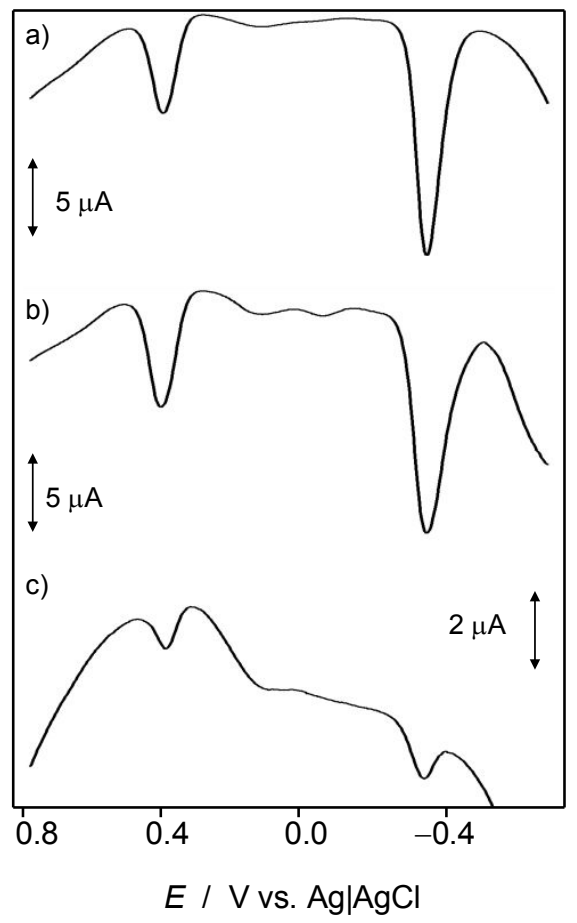


Figure S.5. Square wave voltammograms of a) indigo, b) IND@ $\mathrm{PAL}_{160}$ specimen (5 $\mathrm{wt} \%$ indigo), and c) IND $@ \mathrm{PAL}_{160}$ specimen (10 wt $\%$ indigo) attached to graphite electrodes in contact with $0.25 \mathrm{M} \mathrm{HAc} / \mathrm{NaAc}$ aqueous acetate buffer at $\mathrm{pH} 4.75$. Potential scan initiated at $-0.75 \mathrm{~V}$ vs. $\mathrm{Ag} / \mathrm{AgCl}$ in the positive direction; potential step increment $4 \mathrm{mV}$; square wave amplitude $25 \mathrm{mV}$; frequency $5 \mathrm{~Hz}$. The dotted arrow mark the peak splitting recorded in the oxidation process at ca. $0.45 \mathrm{~V}$.

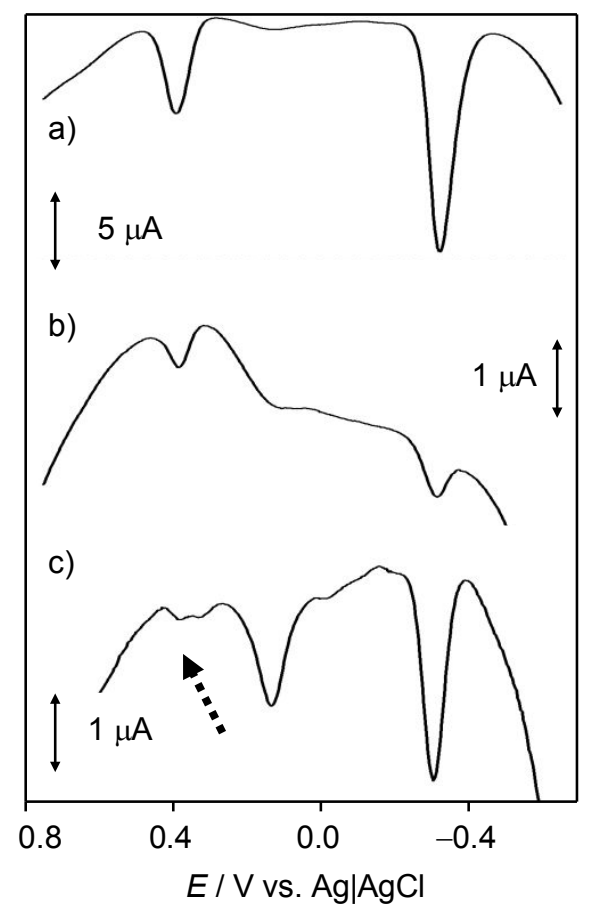


Figure S.6. Plots of $\Delta G_{\mathrm{TAU}}$ vs. $T$ from UV-Vis spectral data in this study represented in Figure 11a, as described in Eqs. (4)-(6).

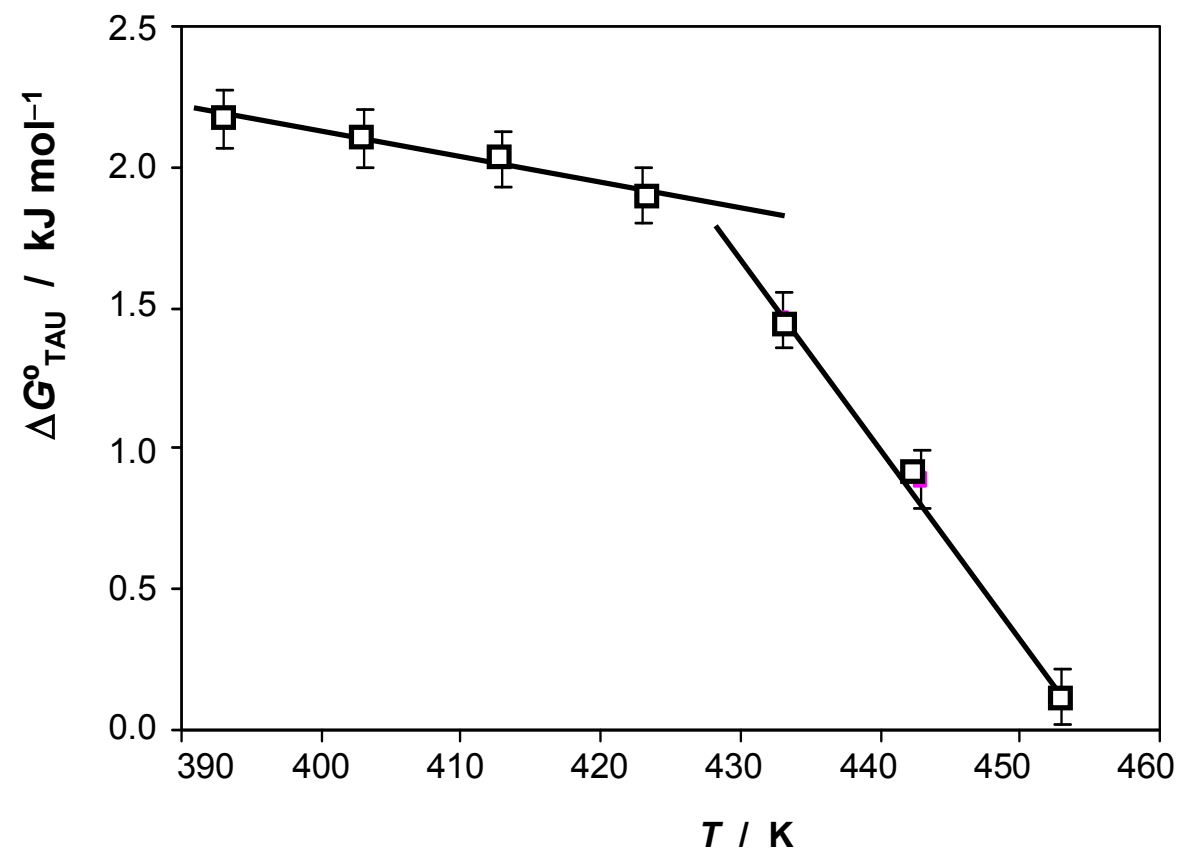


Figure S.7. Plots of $\Delta G_{\mathrm{TAU}}$ vs. $T$ from Raman spectral data reported by De Faria et al. ${ }^{35}$ (see Figure 11b), as described in Eqs. (7)-(9).

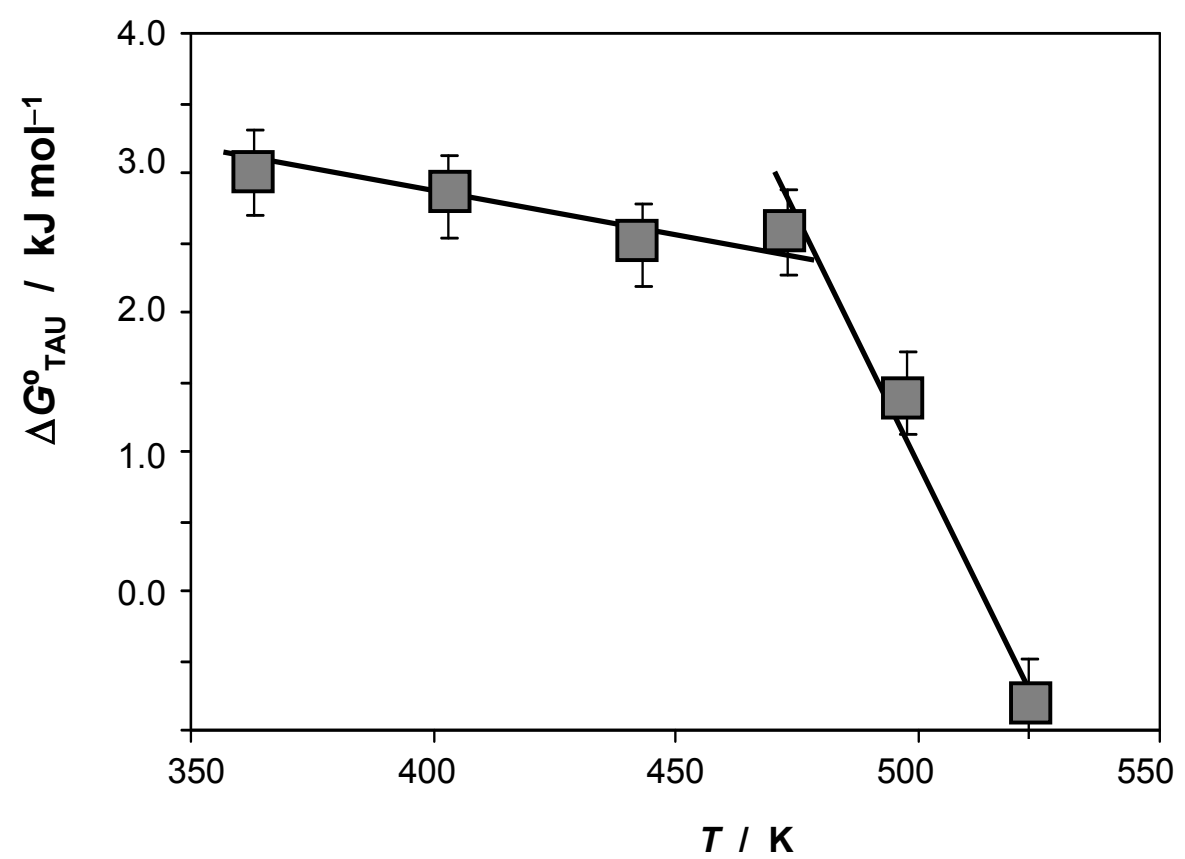


Table S.1. ${ }^{13} \mathrm{C}$ NMR spectrum of IND@SEP ${ }_{180}(10 \mathrm{wt} \%$ indigo $)$ specimen) and reported ${ }^{13} \mathrm{C}$ NMR spectra of indigo (IND), dehydroindigo (DHI), IND@SEP specimens.

\begin{tabular}{|c|c|c|c|c|c|}
\hline Assignment & IND $^{[5]}$ & DHI ${ }^{[17]}$ & $\begin{array}{l}\text { IND@ } \\
\text { SEP }\end{array}$ & $\begin{array}{l}\text { IND@ } \\
\text { SEP }^{[32]}\end{array}$ & $\begin{array}{l}\text { IND@ } \\
\text { SEP } \\
\text { This work }\end{array}$ \\
\hline \multirow[t]{4}{*}{$\mathrm{C} 3(\mathrm{C}=\mathrm{O})$} & & & & & 193 \\
\hline & 191.1 & & 191.4 & 191 & 191 \\
\hline & & 189.5 & 189.3 & 189 & 189 \\
\hline & & & $(186)$ & & \\
\hline $\mathrm{C} 2$ & & 159.5 & & & \\
\hline C9 & & 155.2 & & & 155 \\
\hline $\mathrm{C} 8$ & 153.3 & & 153.3 & 153 & 153 \\
\hline $\mathrm{C} 8$ & & 139.1 & & & 139 \\
\hline $\mathrm{C} 6$ & & 136.8 & & & 136 \\
\hline $\mathrm{C} 4$ & & 130.4 & & & \\
\hline $\mathrm{C} 5$ & & 125.1 & & & \\
\hline $\mathrm{C} 7$ & & 124.2 & & & \\
\hline \multirow[t]{2}{*}{ C6 } & 137.8 & & 138.3 & 136 & 138 \\
\hline & & & & 134 & \\
\hline $\mathrm{C} 2, \mathrm{C} 4, \mathrm{C} 5, \mathrm{C} 7$ & $\begin{array}{l}125.2 \\
124.0 \\
122.1 \\
120.7\end{array}$ & & 122.2 & $\begin{array}{l}124 \\
122 \\
120\end{array}$ & $\begin{array}{l}126 \\
125 \\
124 \\
122 \\
121 \\
120\end{array}$ \\
\hline C9 & 113.3 & & 114.1 & 114 & 114 \\
\hline
\end{tabular}

